Cinémas

Revue d'études cinématographiques

Journal of Film Studies

\title{
Le paysage dans les vues Lumière
}

\section{Marco Bertozzi}

Volume 12, numéro 1, automne 2001

Le Paysage au cinéma

URI : https://id.erudit.org/iderudit/024865ar

DOI : https://doi.org/10.7202/024865ar

Aller au sommaire du numéro

\section{Éditeur(s)}

Cinémas

\section{ISSN}

1181-6945 (imprimé)

1705-6500 (numérique)

Découvrir la revue

Citer cet article

Bertozzi, M. (2001). Le paysage dans les vues Lumière. Cinémas, 12(1), 15-33. https://doi.org/10.7202/024865ar

\section{Résumé de l'article}

L'idée que la tradition du « védutisme » est visible jusque dans les vues des frères Lumière rattache leur production aux séries culturelles qui leur sont antérieures, discréditant ainsi le mythe rassurant de l'invention improvisée. La volonté de représenter du " pays » implique par conséquent une confrontation à une vaste conception historiographique dans laquelle, au-delà de l'art impliqué (peinture, scénographie, photographie) ou du dispositif utilisé (chambre noire, lanterne magique, mondo niovo, stéréoscope), des modèles forts dominent la scène. Alors que la ville et ses monuments sont devenus des clichés, sous forme de cartes postales pour le touriste, le cinématographe Lumière édifie de nouveaux imaginaires urbains, en posant une question à la nouvelle historiographie : ces paysages sont-ils des " paysages d'auteur "? d'utilisation que vous pouvez consulter en ligne.

https://apropos.erudit.org/fr/usagers/politique-dutilisation/ 


\title{
Le paysage dans les vues Lumière
}

\section{Marco Bertozzi}

\begin{abstract}
RÉSUMÉ
L'idée que la tradition du "védutisme" est visible jusque dans les vues des frères Lumière rattache leur production aux séries culturelles qui leur sont antérieures, discréditant ainsi le mythe rassurant de l'invention improvisée. La volonté de représenter du "pays» implique par conséquent une confrontation à une vaste conception historiographique dans laquelle, au-delà de l'art impliqué (peinture, scénographie, photographie) ou du dispositif utilisé (chambre noire, lanterne magique, mondo niovo, stéréoscope), des modèles forts dominent la scène. Alors que la ville et ses monuments sont devenus des clichés, sous forme de cartes postales pour le touriste, le cinématographe Lumière édifie de nouveaux imaginaires urbains, en posant une question à la nouvelle historiographie: ces paysages sont-ils des "paysages d'auteur"?
\end{abstract}

\begin{abstract}
The idea that a landscape tradition is visible in Lumiere films links their work to pre-existing cultural series, far removed from the reassuring myth of extemporaneous inventiveness. Representing the country (side) means confronting an immense historiographic tradition in which, regardless of the art form (painting, stagecraft, photography) or technology used (camera obscura, magic lantern, mondo niovo, stereoscopy), forceful elements served as models. While tourist postcards had already stereotyped the city and its monuments, the Lumière cinématographe constructed a new urban imaginary by adding a new line of inquiry to the new historiography: are those landscapes "authored landscapes?"
\end{abstract}




\section{Temps, vue, résistance}

En abattant les temples de l'histoire panthéon, la nouvelle historiographie du cinéma adopte une conception dynamique de la visualité et, par-là même, revient à l'inertie propre au goût "védutiste ${ }^{1}$ ". Pour l'« icononaute ${ }^{2}$ ", l'appartenance à un marché des imaginaires en quelque sorte déjà structurés, et dont les racines profondes déterminent son cheminement visionnaire, lui assure ses entrées dans l'art voluptueux du cinématographe. Les imaginaires, qui ont été étendus lors de leurs "traversées" de chambres noires ou agrandis dans le faisceau des lanternes magiques, qui ont été scrutés dans un Mondo niovo ou dans un Megatelescopio, observés au Panorama ou au Cosmorama, vont jouir désormais d'une multitude d'effets de réalité garantis par le cinématographe. Apparaît sur l'écran la matérialisation, une autre matérialisation, du système signifiant de l'espace; par ses dimensions gigantesques, le tableau devient paysage et contient, par conséquent, des règles et des modèles, des pratiques et des passions inhérentes à la «naturalité» de la vue.

La vue Lumière scrute certes les anciens thèmes — par tradition -, mais rompt l'appartenance à la perspectiva artificialis immobile du "modèle italien ": ou mieux encore, en maintient le tableau et la composition mais en change les dimensions, la temporalité et la jouissance. La particularité du paysage "cinédynamique» repose alors sur une tension visuelle hyperbolique engendrée par l'illusion macroscopique d'un mouvement continu et régulier. Si, dans un premier temps, on suppose une certaine continuité thématique avec les représentations antérieures, la ville, lieu par excellence de flux d'échanges, d'énergies et de dynamismes, devient le sujet cardinal du nouveau dispositif: dans le catalogue Lumière, elle est le sujet de prédilection des vues, le paradigme d'une géographie sentimentale où la stratégie commerciale et la poésie du mouvement engendrent un écheveau d'appartenances que l'on peut difficilement repérer avec les outils théoriques des disciplines historico-cinématographiques traditionnelles. L'idée de "vue" implique en fait une mise en image du paysage - naturel comme urbain — qui n'a rien de moderne, malgré la modernité du dispositif; l'idée de vue nous fait rattacher cette mise en image à un acte antique et à des hori- 
zons antérieurs de la vision. Or, les Lumière se sont intéressés à la ville plus qu'au paysage de la nature (souvent immobile, si on excepte les ressacs lyriques des vagues moussantes ou l'agitation des feuillages secoués par les vents). La ville est l'espace de composition photographique par excellence, l'icône du progrès, le théâtre d'échanges et de représentations sociaux, le lieu des premières dynarnisations de la caméra. La ville est également présente en raison de la passion qu'elle inspire depuis plusieurs siècles. Comme l'avance De Seta (1982, p. 133 - notre traduction) :

[...] le fait d'identifier le concept de Beau avec la magnificence de la ville, ou de recourir à l'analogie entre les deux, est un phénomène qui acquiert un rôle important dans la sensibilité esthétique de la culture européenne au seuil de l'âge moderne.

Cette attitude persiste clairement dans la production Lumière et justifie la plupart des vues du catalogue. Une part des particularités du paysage d'autrefois émergent dans la liste des titres. Les principaux attributs de l'architecture européenne imprègnent l'iconographie Lumière, à savoir : la stratification des villes historiques - encore assez évidente au tournant des XIX et $\mathrm{XX}^{\mathrm{e}}$ siècles -, les dettes topographiques envers les installations médiévales, le recours typologique aux places, églises, portes, remparts: voici, dans le catalogue Lumière, que l'on trouve l'Arc de triomphe $\left(\mathrm{n}^{\circ} 149\right)$ ou le Panorama de la place Saint-Marc pris d'un bateau ( $\left.\mathrm{n}^{\circ} 296\right)$, la Porte de Tolède ( $\mathrm{n}^{\circ} 261$ ) ou la Karlsplatz de Munich (n'235).

Alors que les Lumière mettent au point le cinématographe, Elisée Reclus, auteur de l'imposante Nouvelle Géographie universelle et rédacteur de guides sur la France pour les Éditions Joanne, soutient que "là où grandissent les cités, l'humanité progresse, là où elles dépérissent la civilisation elle-même est en danger " (Reclus, cité dans Paquot et Roncayolo, 1992, p. 159³). Le catalogue Lumière semble bâti sur cette idée. Paris est, par antonomase, la ville "mise en scène"; les syntagmes les plus représentatifs de Paris sont alors ceux de la ville moderne comme les «boulevards" (l'expression est du couple Napoléon 
III/Préfet Haussmann), que l'on retrouve sous deux versions différentes dans Paris: Bassin des Tuileries $\left(\mathrm{n}^{\circ} 150\right)$ ou Paris: Champs-Élysées ( $\left.\mathrm{n}^{\circ} 151\right)$. Et pourtant les Lumière semblent, en même temps, conscients de l'effet dynamique limité que leur offrent les œuvres monumentales ou les grands restes du passé. D'ailleurs, si on considère la rareté de ces thèmes dans les fresques védutistes les plus dynamiques, on est amené à supposer un intérêt privilégié pour le tableau d'une ville perçue comme cours et scènes, plutôt civitas - villes des hommes - que urbs ville de pierre. André Gardies (1995, p. 13) soutient que:

\begin{abstract}
[...] l'architecture ne constitue pas un objet d'élection donc ne vaut pas pour elle-même, elle est en revanche nécessaire car elle a valeur de signes. Elle est d'abord la marque du génie humain, la marque de l'homo faber et de son pouvoir inventif.
\end{abstract}

C'est la «machine ville» non pas «le temple de l'art» qui passionne les opérateurs Lumière. Ainsi, une vue du Parvis de Notre-Dame $\left(\mathrm{n}^{\circ}\right.$ 691) n'a rien de comparable aux évocations littéraires de Victor Hugo dans Notre-Dame de Paris ou aux attentions amoureuses historico-architecturales de la restauration de Viollet-le-Duc. Le monument résiste au cinéma et engage dès le départ, une bataille avec le film, un corps à corps dont l'enjeu est le sens et la prédominance d'un texte - architectural ou filmique - sur l'autre. C'est tout le problème de la traduction intersémiotique entre les deux arts: l'œuvre architecturale peut se plier aux structures de sens mises en place par le texte filmique mais peut également s'y opposer, en maintenant l'autonomie expressive, quoique reprise et resémantisée, que lui confere sa capacité historico-stylistique à se faire reconnaître. Ce n'est que partiellement réinvesti par une nouvelle signification cinématographique que le monument vu au cinématographe supporte la "lourdeur " de sa célébrité : cinquante secondes, ce n'est pas grand-chose pour visiter le Colisée, le Louvre ou la cathédrale de Saint-Paul; cinquante secondes, c'est bien long pour le spectateur intéressé au flux d'images animées du cinématographe quand il les voit se figer comme glace sur le visage inerte et glorieux du passé. Question de stéréotypes? 


\section{Paysages et stéréotypes}

Dans le village de Namo (cf. Panorama pris d'une chaise à porteur, $\mathrm{n}^{\circ} 1296$ ), l'opérateur, qui a rivé l'œil cyclope du cinématographe à ras de terre, sur la chaise à porteur, et qui filme l'aveuglante nudité d'un enfant courant vers la caméra, donne l'impression de fuir, de vouloir sortir de ce stéréotype. C'est dire que la modernité de certaines vues est (devait être) insoutenable. Pourtant, d'un autre côté, la construction du progrès colonial va de pair avec l'édification des villes. Alors que les cabanes du peuple de Namo restent l'archétype d'une architecture d'origine, les paysages exotiques des Lumière reposent en fait fréquemment sur des scénarios architecturaux occidentaux, et apparaissent comme les témoins du processus de "développement" en cours. Même au Japon, l'opérateur Constant Girel choisit de placer les deux Lutteurs japonais $\left(\mathrm{n}^{\circ}\right.$ 925) de Kyoto devant un temple qui semble une copie, en miniature, de la Bourse de Bruxelles; les rues embouteillées, les marchés, les casbahs des villes arabes nous rappellent le grouillement du Moyen Âge sans loi - et sont bien loin de représenter le Moyen Âge galant reconstruit, lui, dans le Vieux-Paris, pour l'Exposition universelle de 1900.

Ainsi, avec l'arrivée du cinématographe, la multiplication des paysages loirıtains amplifie l'exercice généalogique de la perspective évolutionniste et ethnocentriste, révélant du même coup une incompréhension structurelle qui consiste à expliquer exotiquement la différence en en consommant l'image. Le regard critique qui interroge l'utilité du savoir, ses buts et ses bénéficiaires est absent du mythe positiviste du progrès. La Place du Gouvernement $\left(\mathrm{n}^{\circ} 200\right)$ comme la Place de l'Opéra ( $\left.\mathrm{n}^{\circ} 372\right)$ ou la Rue de France ( $\mathrm{n}^{\circ}$ 205), ce sont Alger, Le Caire, Tlemcen imaginés par Paris, des toponomastiques d'un urbanisme miroir et moteur du développement, promptement reprises par les opérateurs Lumière.

Le risque de travailler sur le stéréotype a été plusieurs fois souligné : l'opérateur privilégie des modèles reconnaissables sans pour autant avoir un projet iconographique bien clair et la communication cinématographique perpétue les topos d'une imagerie touristique que structurent des systèmes médiatiques plus larges. La représentation de l'Italie, par exemple, malgré une 
centaine de vues qui l'évoquent, s'avère absolument fragmentaire. Alors que le rideau tombe sur l'époque romantique du voyageur et que s'instaure celle, plus rapide, du touriste, les villes de Turin, Milan, Monza, Gênes, Venise, Carrare, Livourne, Florence, Rome, Naples, Pompéi, Castellamare di Stabia, Cagliari, Sienne (avec prises de vues du Palio, hors catalogue) sont filmées par les opérateurs Lumière sans un projet illustratif général. D'importants sites monumentaux - Venise, place Saint-Marc (Pigeons sur la place Saint-Marc, $\mathrm{n}^{\circ}$ 292) alternent avec des itinéraires romains et florentins filmés négligemment et de façon expéditive, tandis que la plupart des vues turinoises sont extrêmement «institutionnelles" — commémorations, visites, inaugurations.

L'Italie provinciale et paysanne du début du siècle n'apparaît pas dans ces vues, le pays "vu d'en bas", tel que le rappelle Piero Camporesi - "un pays observé à partir d'une boutique, d'une place, d'un champ, d'une taverne, d'un réfectoire de couvent" (1992, p. 13) —, reste une image "inconcevable" aux yeux des Lumière. Les films de leurs opérateurs font plutôt ressortir une Italie qui, sur la base des valeurs de la tradition monarchique, assiste à la (présumée) modernisation de sa culture. Entre le XIX et le $\mathrm{XX}^{\mathrm{e}}$ siècle, le stéréotype du paysage se transmet par la carte postale illustrée, les objets-souvenirs comme la colonnade de Bernin ou la tour Eiffel, les affiches publicitaires des compagnies ferroviaires et transatlantiques, les revues illustrées ou les guides de voyages ${ }^{4}$. Jacques Aumont rappelle que "la vue Lumière partage avec la carte postale le souci de faire tableau, de cadrer la réalité pour l'isoler à l'état d'événement, bref de donner d'emblée une vue complète» (Aumont, 1984, p. 33). À l'intérieur du système médiatique, on note également des correspondances entre les maisons d'éditions touristiques et le cinéma naissant. En feuilletant le guide Joanne consacré à Venise - parmi de nombreuses curiosités, on mentionnera celle du tempo touristique de l'époque: "huit jours au moins sont nécessaires pour visiter Venise et en prendre une idée suffisante" (1895, p. 22) - , on peut faire des rapprochements saisissants entre les paysages décrits dans le guide et les vues contemporaines des Lumière tournées sur la lagune. 
Pigeons sur la place Saint-Marc ( $\mathrm{n}^{\circ}$ 292) montre une dame vêtue de blanc qui offre des grains aux pigeons en vol. Un homme entre dans le tableau par le côté droit, agite les bras pour inciter les volatiles au mouvement, pendant que la femme s'approche lentement de la caméra: l'homme persiste à animer la scène, en passant derrière la femme et en se plaçant au centre $\mathrm{du}$ tableau. Les pigeons continuent leur tourbillon. Ils sont, pour en revenir au guide Joanne (1895, p. 22), cette

\begin{abstract}
[...] classe d'habitués de la place Saint-Marc qu'il ne faur pas oublier... C'est un curieux spectacle de voir cette foule aérienne accourir et s'abattre de toutes parts, quand l'horloge frappe les coups de 2 heures, moment quils savent être celui de la distribution des grains qui leur est faire.
\end{abstract}

Au-delà de la "couleur locale", un lien iconographique plus étroit peut être fait avec le guide Joanne. À la page 25, la seule et unique photographie de la publication représente la basilique Saint-Marc, vue de la place d'en face ${ }^{5}$. Observons la façade filmée symétriquement et encadrée par les hampes de quatre drapeaux. À mi-chemin entre le point de reprise et la façade, exactement au centre de la composition, un groupe de personnes profite du soleil. La vue Venise: place Saint-Marc $\left(\mathrm{n}^{\circ} 430\right)$ semble être, sans métaphore, une photographie animée du même objet. Identique dans la composition, dans la distance focale des trois plans principaux de la "scène" - façade, hampes, personnes -, elle ne se différencie que par l'entrée dans le tableau de deux "animateurs" qui, après avoir traversé le champ de droite à gauche tout en bavardant vivement entre eux, comme pour nous confirmer le caractère "insoutenable» de la vue monumentale, vont se confondre avec le petit groupe de personnes au centre de la vue.

On peut en déduire que le carnet du voyageur de la fin du $\mathrm{XIX}^{\mathrm{e}}$ siècle s'est enrichi de valeurs artistico-paysagères canonisées, c'est-à-dire de stéréotypes touristiques suffisamment structurés pour offrir les mêmes représentations conventionnelles de monuments architecturaux célèbres. La place Saint-Marc est justement, depuis des siècles, un lieu de spectacles et de 
représentations védutistes. Au XIX siècle, le recours à des représentations analogues à celle de la prise de vue Lumière et de la photographie du guide Joanne se retrouve dans: 1) la gravure à daguerréotype de la collection Costantini (1841); 2) la photographie tirée de l'Album photographique de l'artiste et de l'amateur des éditions Blanquart-Évrard $(1851)$; 3) une gravure à l'albumine de Carlo Ponti (vers 1860) ; 4) une photographie d'Achille Quinet (1865). Même le célèbre conférencier John L. Stoddard représente Saint-Marc du même point de vue, pour un de ses albums photographiques de la fin de siècle ${ }^{6}$. Au début du $\mathrm{XX}^{\mathrm{e}}$ siècle, dans un article sur les «Nouvelles Conférences avec projections ", Le Fascinateur rapporte la présence d'une photographie analogue: "voici la basilique byzantine de Saint-Marc, la plus intéressante des quatre-vingts églises de Venise" (anonyme, 1904, p. 332).

Mais cette perspective n'empêche pas une autre hypothèse. Est-il possible que l'opérateur français, en arrivant sans aucun support informatif et animé seulement par un imaginaire vénitien stéréotypé, aille se placer exactement au même endroit que celui illustré sur la photographie du guide Venise? Peut-être qu'Alexandre Promio, infatigable globe-trotter ayant parcouru l'Espagne, l'Angleterre, la Belgique, la Suisse, la Turquie, la Russie, l'Italie, l'Allemagne, la Suisse et les États-Unis, utilisait, tout comme beaucoup d'autres opérateurs Lumière, un instrument riche de conseils et d'informations expéditives — sans parler de la littérature du Grand Tour, culturellement éloignée de l'esprit des opérateurs - , qui permettait de rapides "immersions" dans les beautés de la lagune. "En trois semaines, avec notre cinématographe, nous avons possédé le monde entier" (cité dans Pinel, 1974, p. 439), aimait répéter Louis Lumière. Sans même l'aide d'un petit guide?

\section{Vers le panorama}

La visite de Promio à Venise correspond à un épisode inscrit dans la légende des origines: le premier travelling de l'histoire du cinéma. L'idée de l'opérateur était que "si la caméra immobile permet de reproduire des objets mobiles, on pourrait peut-être retourner la proposition et essayer de reproduire à l'aide du 
cinéma mobile, des objets immobiles " (Promio, cité dans Coissac, 1925, p. $197^{7}$ ). Dans la vue Panorama du Grand Canal pris d'un bateau, Promio filme le Grand Canal d'un traghetto en marche - version plus vraisemblable que celle de la prise de vues en gondole. Il inscrit sur l'eau la merveilleuse séquence des édifices dans un parcours dynamique, semblable au Prospectus Magni Canalis Venetiarum de Canaletto, grand védutiste du XVIII ${ }^{e}$ siècle. Le cinématographe permet cette vision panoramique que le védutiste vénitien obtenait lorsqu'il faisait se côtoyer, tout en les réélaborant, ses scaraboti (gribouillages) obtenus à l'aide d'une chambre noire: même processus de capture optique de l'image - dans les deux cas il s'agit de chambre noire -, bien que la modalité d'impression du support soit différente - manuelle dans un cas, photochimique dans l'autre -, mêmes analogies suggestives sur la temporalité de la restitution/jouissance. La multiple acception de points de vue ${ }^{8}$ permet d'en définir de façon préliminaire le dynamisme commun. Que ce soit l'expérience de Canaletto ou le panorama de Promio, ils demandent tous les deux le mouvement: mais si, dans le premier cas, le point de vue de l'observateur est impliqué, c'est à l'œil de se déplacer, en saisissant des séquences spatio-temporelles successives où sont comprises de nouvelles portions du filmage - dans le deuxième cas, le panorama de Promio, c'est au point de vue observé, c'est-à-dire à la vue même, de rendre le "mouvement " de la cour architecturale sur le Grand Canal.

Le rapide développement du tourisme naissant, l'acquisition et l'occidentalisation de territoires coloniaux, la modernisation industrielle des pays d'Europe centrale semblent avoir été scrutés, contemplés, recherchés dès les premiers panoramas Lumière. La caméra explore les possibilités d'un point de vue quelconque, en le réduisant en mille pièces, en le révoquant peut-être. En se plaçant au-dessus, au-dessous, à l'intérieur, derrière, devant les moyens de transport de la civilisation à vapeur, elle amplifie l'irréductible et fantastique captation réaliste du monde. Comme l'écrit Philippe Roger (1999, p. 338):

Rendue solidaire d'un moyen de transport, la caméra des panoramas ne fait que vérifier la continuité du monde déjà impliquée par la vue fixe. La beauté des 
panoramas est de l'ordre de la confirmation: dans l'unité retrouvée du monde, l'espace se montre ouvert, pluriel; la continuité d'un monde ouvert se conjugue à la pluralité des lieux.

Depuis la locomotive d'un train en marche jusqu'au tunnel qui engloutit la vision, nous voyons défiler un faisceau de rails encastrés - Passage d'un tunnel en chemin de fer (pris de l'avant de la locomotive); puis l'œil de la caméra observe des activités et des architectures du bord de Seine - Panorama de rives de la Seine, I, II, III, IV (n $\mathrm{n}^{\text {os }} 684,685,686,687$ ); s'attarde, depuis la tête d'un tramway, sur un décor urbain bien ordonné - Montreux. Panorama ( $\left.\mathrm{n}^{\circ} 1135\right)$; balaye le paysage industriel des docks de Liverpool, en traversant les places de la Great Western Railway ou les dépôts de la Manchester Ship Canal - Panorama pris du chemin de fer électrique, III ( $\mathrm{n}^{\circ} 706$ ); détaille, depuis un bateau sur la Seine, la variété de styles des pavillons nationaux de la Grèce, de la Suède, de Monaco, de l'Espagne, de l'Allemagne, de la Norvège, de la Belgique, de la Grande-Bretagne, de la Hongrie, de la Bosnie-Herzégovine, de l'Autriche, des ÉtatsUnis, de la Turquie et de l'Italie, montés pour l'Exposition parisienne de 1900 - Rue des Nations, I, II, III ( $\mathrm{n}^{\text {os }} 1163$, 1164, 1165). Grâce au mode panoramique, l'interpénétration entre vision filmique et paysage devient multiple. C'est la dernière étape de l'observation réaliste du monde: la modalité du regard intense et analytique du voyageur moderne s'oppose à l'approche symbolique et surnaturelle du pélerin médiéval, que n'intéressent, mises à part leurs résonances religieuses, ni le paysage naturel ni les remparts de l'urbs. Le panorama Lumière est fils de l'opérateur errant et de son plaisir à s'aventurer dans le physique des lieux filmés; sa soi-disant flânerie de l'œil est accentuée, une dimension ultérieure multiplie la représentation au carré - le mouvement de la foule, le mouvement des images - déjà garantie par le filmage fixe. Voici une modalité de perception semblable à la sublime décision de The Man of the Crowd d'Edgar Allan Poe: sortir de l'endroit d'où l'on observe immobile la vie en ville, abandonner le faisceau de regards immobiles de ce seul et unique point de vue, pour s'immerger - au-delà des vitrines dans un bain de foule de la ville Lumière. 


\section{La ville scénique}

En dehors de sa propre mise en scène, où il fait valoir sa beauté et son autonomie esthétique, le paysage urbain devient également le plateau de scène original de spectacles, de numéros qui valent pour eux-mêmes, mais qui se trouvent enrichis par le décor urbain qui les accueille. Tout d'abord, des performances comiques: les désastres du Photographe introspectif $\left(\mathrm{n}^{\circ} 118\right)$ comme le vacarme plumeux de la Querelle des matelassiers ( $n^{\circ} 948$ ); les vues comiques du Faux Cul de jatte (n" 665) - un faux invalide s'enfuit à l'arrivée du policier - ou du Cocher endormi ( $\mathrm{n}^{\circ}$ 666) on remplace le cheval d'un cocher par un cheval de bois à bascule; les petites pièces clownesques comme Chapeaux et équilibres $\left(\mathrm{n}^{\circ} 463\right)$ ou Le Gendarme et le voleur ( $\left.\mathrm{n}^{\circ} 467\right)$, toutes tournées en plein air, dans un contexte urbain. Même plateau "nature" pour des pas de danse comme dans la Danseuse des rues à Londres $\left(\mathrm{n}^{\circ} 249\right)$ ou dans la Danse des Ciociari à Rome ( $\left.\mathrm{n}^{\circ} 289\right)$, pour la Course en sac $\left(\mathrm{n}^{\circ} 109\right)$ et les plongeons aux Bains de Diane $\left(\mathrm{n}^{\circ} 277\right)$ à Milan, pour l'atmosphère romantique de la Bataille de neige ( $\mathrm{n}^{\circ} 101$ ) ou pour les défilés du Carnaval parisien dans le Cortège du bouf gras (char du prince carnaval) ( $\left.\mathrm{n}^{\circ} 155\right)$.

De même que la tradition védutiste antérieure soulignait un parcours fécond entre villes et théâtres, la vue Lumière saisit la spectacularisation de l'urbs dans ses multiples possibilités'. Des défilés militaires aux processions religieuses, des fêtes civiles jusqu'à un échantillon d'actions interprétables selon le modèle de la "vie quotidienne comme représentation" - se montrer, saluer, se présenter, arriver, partir, monter, descendre, circuler, travailler, photographier, etc. - la "ville scène» accueille les gestes d'une majestueuse récitation collective. L'analyse des niveaux de représentation dans la "ville scène" fait ressortir un phénomène intéressant. Quand l'action sort du théâtre de pose, le point de vue instauré par l'opérateur fait tomber la prétendue «théâtralité» catégorie utilisée pour définir la frontalité de la prise de vues dans le cinéma des origines, et qui mériterait une plus grande analyse - pour se ranger sur les conseils des Lumière à propos de la composition des sujets photographiques. En d'autres termes, la frontalité et la symétrie des vues comiques, vues historiques ou vues de danse filmées au théâtre ne sont plus garanties pour des sujets 
similaires tournés en ville. Placé de façon excentrique par rapport au croisement des diagonales, le point de vue de l'opérateur témoigne, "en profondeur", de la performance des désinvoltes Danseuses des rues à Londres: la route mouillée par la pluie, les enseignes des locaux, le public improvisé, les danseuses qui risquent constamment elles aussi de sortir du tableau, montrent l'écart existant avec les vues du Ballet de Flora ( ${ }^{\text {os }}$ 904-908), une série où les danseuses filmées occupent le centre de l'image et se produisent selon une scénographie symétrique. Les mêmes analogies, les mêmes différences peuvent être remarquées entre les Nègres dansant dans la rue $\left(\mathrm{n}^{\circ} 252\right)$ et la série du Ballet Excelsior, du $\mathrm{n}^{\circ} 909$ au $\mathrm{n}^{\circ} 913$. Les petites pièces clownesques de la vue $n^{\circ} 460$ à la vue $n^{\circ} 467$, tournées quant à elles dans la rue, sont régulièrement filmées en diagonale, alors que de semblables performances de jongleur, dans les vues 898-903, mises en scène dans un décor théâtral, reviennent au point de vue central.

La division n'est pas toujours aussi nette. Quelquefois peut-être quand le poids du syntagme urbain est faible? - le point de vue hésite entre les deux modèles: c'est le cas des Danses espagnoles ( $\mathrm{n}^{\circ}$ 843-854), filmées de différents points de vue devant une archivolte mauresque de Séville; c'est aussi le cas des vues comiques mises en scène dans des décors postiches et filmées diagonalement dans L'Ivrogne $\left(\mathrm{n}^{\circ} 884\right)$ et de face dans L'Amoureux dans le sac ( ${ }^{\circ}$ 885). Quand il y a recherche de mise en scène de personnages, le poids spécifique de la "ville scène" prévaut quand même sur celui du plateau théâtral. Le cinématographe privilégie la symbiose dynamique - encore que métonymique - avec le mouvement renfermé entre les remparts de l'urbs. Élément fondateur du catalogue, la ville instaure des modalités représentationnelles fortes, où la profondeur de champ, loin de constituer une avant-garde inexplorée, selon un des paradigmes internes au débat du classicisme, semble plutôt continuer la tradition photographique du XIX ${ }^{\mathrm{e}}$ siècle selon laquelle le sujet filmé devait être parfaitement net ${ }^{10}$.

\section{Impressionnismes}

L'iconographie Lumière s'insère très librement dans la lignée des représentations impressionnistes de la ville. Après que 
Claude Monet ait illustré les flots vaporeux de la Gare SaintLazare, que Gustave Caillebotte ait "photographié", avec un étonnant réalisme, un instant quelconque sur le Pont de l'Europe et que Pissarro ait peint les grouillants décors du nouveau Paris comme l'Avenue de l'Opéra ou le Boulevard Montmartre", le paysage Lumière apparaît alors qu'on mène une réflexion sur la quantité et sur la qualité des effets de réalité et sur les problèmes posés par les limites du tableau, notamment au regard des parcours de "libération du regard " qu'a connus le XIX siècle, dans des sphères médiatiques plus larges que la seule sphère cinématographique. À propos de ces années, Antonio Costa (1981, p. 341 - notre traduction) rappelle que:

[...] le "paysage" de la tradition picturale renvoie, de façon programmatique, "au-delà de la peinture", il rentre dans le domaine de la consommation de masse en tant qu'élément d'attraction, à côté d'autres attractions comme l'illustration journalistique, l'aménagement urbain, la marchandise spectacularisée dans les expositions universelles, les curiosités scientificotechnologiques, les "machines", etc.; et l'attraction du contenu même de la vision est complémentaire à celle de la technique qui la rend possible.

Certes, entre le cinéma naissant et la peinture impressionniste, il existe bien quelques analogies. Comme l'impressionnisme, le cinématographe est attiré par le plein air. Pratique, léger, réversible, il se concentre sur la "vibration" de la foule pour saisir des séries véridiques d'instants prégnants, des dynamismes changeants de la vie en ville, des impressions, des nuages et des fumées qui modifient de façon irréversible le système de perception de l'observateur/spectateur. Il s'agit d'une épaisseur sensorielle raffinée, garantie par l'apparence réaliste du fugitif, allant de la fumée d'une cigarette à la mousse d'un demi de bière, du scintillement d'un bouton au flottement d'un drapeau. Autre point de rencontre entre le cinématographe et l'impressionnisme, corollaire du précédent, est le fait que l'espace urbain s'avère déstabilisé par l'évocation sensible du sujet/point de vue: «une scène précaire qui est le fruit d'une focalisation variable comme celle du futur plan du cinéma et qui délie ce qui reste 
des règles compositionnelles et des cadrages classiques de leurs symbolismes figés" (Natali, 1996, p. 60); ou bien, dernière ressemblance, la non-représentation des secteurs de la ville qui avaient été négligés par les transformations haussmanniennes et posthaussmanniennes. Même si la vue Lumière semble «spirituellement" apparentée au sentiment des impressionnistes, on s'aperçoit que les références à tel ou tel autre auteur restent des prétextes pour de faciles correspondances formelles. On jugera donc hasardeuse l'institution d'associations iconographiques entre le védutisme des Lumière et les ouvres de Cézanne, Manet, Monet, Pissarro, Renoir. On estimera déplacée l'idée que les itinéraires des opérateurs se soient faits sur les traces du Grand Tour et sur celle de la littérature en question. La provenance culturelle de nombreux opérateurs rend encore plus improbable un citationnisme conscient et voulu.

Dans "Usines, faubourgs et prise de conscience ouvrière", Laurent Gervereau (1994) remarque une tendance de la peinture urbaine, entre le $\mathrm{XIX}^{\mathrm{e}}$ et le $\mathrm{XX}^{\mathrm{e}}$ siècle, à présenter comme rassurante l'intrusion violente de la révolution industrielle. Les paysages suburbains des banlieues, avec leurs cheminées fumantes, les murs aveugles des usines, les quartiers surpeuplés, apparaissent presque comme des accessoires naturels de décoration. Lurbain industrialisé n'entame pas le paysage bien établi d'une campagne anthropomorphisée ni le panorama ancestral sur une nature luxuriante. Ces stylèmes n'embrassent pas seulement la peinture inpressionniste: l'harmonie entre les activités de l'homme et les forces de la nature envahissent également le très coloré faubourg de Kandinsky dans Vor der Stadt (1908) ou le radieux plan général de Boccioni dans Officine a Porta Romana ${ }^{12}$. En revanche, les visions de Théophile Alexandre Steilen de 1895 s'avèrent différentes: il décrit un paysage d'usines extrêmement réaliste, encombré de cheminées fumantes, de coups de peintures noirâtres dans un ciel de plomb ${ }^{13}$; ou la représentation de Constantin Meunier, $A u$ pays noir, dans laquelle la perspective se cambre sur une voie ferrée garnie de chariots à charbon, et qui longe une vallée criblée d'usines et de cheminées ${ }^{14}$. Dans ces représentations, il n'y a plus d'espace pour les beautés de la nature: on ne voit ni fleurs, ni arbres, ni animaux, ni hommes. 


\section{Paysages d'auteur?}

Pour imaginer un Louis Lumière auteur, il faut s'efforcer de détourner le regard de l'objet de ses vues et diriger l'enquête vers un scénario beaucoup plus large. Son écrin personnel de "visionnaire» lui permet de scruter des paysages qui apparaissent déjà dotés de solides piliers et capables de supporter les principaux "hiéroglyphes » du continent européen - places, cathédrales, villes - , même en face du dernier et tout nouveau dispositif de la vue dynamique. En fait, face à la nouveauté merveilleuse du cinématographe, on doit admettre la présence constante d'une tradition diffuse, "étalée » sur les longs siècles du "précinéma", constamment rappelée par des spectacles, permanents ou itinérants, d'un auteur collectif encore évanescent; car remémorer, renommer, revoir constituent les termes d'une joie scopique, simple et partageable, nourrie de traces de réel dans "un pacte esthétique intersubjectif qui est le principe de cohésion pour la persistance d'une tradition" (Montani, 1989, p. 649 - notre traduction). Dans la même veine, Gianni Rondolino (1988, p. 55 - notre traduction) soutient que les films-vues Lurnière sont le résultat de:

\section{[...] l'œuvre collective de toute une société dont ils sont le miroir, raison pour laquelle on peut les considé- rer comme des ouvres anonymes, à l'instar de beau- coup d'œuvres littéraires, picturales et architecturales du passé, qui sont le reflet sur le plan de l'art et de la culture d'une certaine conception du monde.}

Plus qu'un auteur, cette perspective fait de Louis Lumière le représentant d'un sentiment commun, un "sentiment vu» où la dentelle des multiples appartenances permet l'affirmation d'une permanence et la reconnaissance d'une espèce, celle de la ville européenne. À l'horizon d'une "auctorialité" faible, on est obligé de se confronter à une multiplication de temps et de perspectives, de contextes et de points de vue capables de porter à la lumière les territoires fuyants de l'identité culturelle et de la réception spectatorielle.

Il s'agit par conséquent d'un plus grand "auteur", d'un «auteur» intinement lié à l'histoire du continent, d'un "auteur» 
qui a élargi de façon démesurée les statut et catégorie de l'antique recherche de l'art comme mimesis. Une intention collective constante au cours des siècles s'incarne dans l'idée même de "vue", dans sa beauté: Aumont (1984, p. 33) rappelle que

\begin{abstract}
[...] dans la vue Lumière, le lieu n'est pas seulement saisi géométriquement, dans son apparente perspective (ainsi qu'a tendance à le faire la photographie de monuments); le lieu filmé est bien un lieu, pas seulement un espace, qui se présente à nous dans son fonctionnement habité.
\end{abstract}

Ainsi, même si les vues Lumière sont toujours bien cadrées et d'une excellente qualité photographique, cela apparaît insuffisant pour faire de Louis le "premier et unique auteur" védutiste cinématographique, ce qui démystifie en quelque sorte la vulgate d'une parthénogenèse artistique où le "maître" semble fondateur d'un genre sans épigones. Un cas éclairant, donc, pour comprendre les processus de création de l'auteur.

Et pourtant, ne sommes-nous pas contre l'idée de voir un auteur en Louis Lumière justement parce que "son idée" n'est et ne peut pas être la vie même prise sur le vif: le désir exprimé par Louis, réitéré par Matuszewski, amplifié par l'antinomie fiction/non-fiction, apparait frustré dès le choix de son point de vue. L'œuvre de Louis met en évidence une puissance révélatrice du visus, c'est-à-dire une forme intuitive capable de donner corps à la réserve disparue des formes précinématographiques (ce qui nous mène bien au-delà des excès emphatiques du biographisme). Par conséquent, si Lumière a retenu, de façon peutêtre excessive, que l'image filmée allait tranquillement du côté du sens pré-vu - un sens clair, limpide, "réaliste», sans traces de résidus herméneutiques -, il a quand même revêtu l'objet extra-cinématographique d'un nouveau langage dont il a inventé les formes linguistico-expressives les plus simples.

Ainsi, les paysages Lumière ne sont presque jamais des images ordinaires, ils ont un poids historico-iconographique et sont témoins de quelque chose de puissant: souvent, ils renvoient à des motifs extra-cinématographiques et, il faut le dire, après que la théorie se soit pourtant démenée à la recherche du spécifique, 
à des motifs qui reviennent et deviennent des sujets, des figures, des transpositions, des flux visuels entre un texte et un autre. Le processus est continu, on a affaire à un recyclage (poétique?) que l'art du cinéma a ultérieurement contaminé et rendu, surtout, mémorable. Entre confluences et influences, la vue Lumière arrive à définir sa propre autonomie, une sorte de code génétique qui en permet l'identification, un cortège de thèses internes à sa forme-écrin. La première de ces thèses est que la ville est le lieu d'élection du cinéma naissant, son berceau effectif et symbolique, le nœud gordien qui enserre la volonté représentative de la maison lyonnaise. Ville comme image vaniteuse, image de concentration dynamique aux vertus ensorcelantes pour le septième art à venir. La seconde affirme que l'ancrage européen influe sur la beauté et la qualité du filmage, garanties "naturellement " par des villes habitées du flux de l'histoire et marquées de la Renaissance au $X X^{\mathfrak{e}}$ siècle par un écheveau imaginaire de séries culturelles différentes, faites de théâtre et de peinture, d'architecture et de littérature. La troisième concerne en revanche les appartenances sociales des Lumière, en raison desquelles on s'éprend d'une certaine ville, de certains fragments - disons-le "institutionnels" - plutôt que d'autres. Une structure à plusieurs niveaux de la vue Lumière apparaît, une structure qui permet de peindre l'autoportrait d'une société. La ville en scène, celle qui est aimée des Lumière, semble un Janus à deux visages: sa matière trompe, la mise à plat de sa représentation sur le plan d'une référentialité apparente et absolue entraîne l'observateur à nier tout aspect imaginatif, cette existence mentale de la ville, qui la constitue avant toute réalité physique. Un dialogue confus s'ensuit, où le mythe du réalisme s'insinue et exclut finalement les éléments de ré-interprétation qui définissent la vue. Les films Lumière comme témoignages "objectifs" de leur époque: tel est donc l'ancien dogme de l'«immaculée perception».

Traduit de litalien par Corinne Baldovini Texte français établi avec le concours de Marion Froger

École nationale du cinéma de Rome Université de Rome III

Le paysage dans les vues Lumière 


\section{NOTES}

1. Nom donné au XVIIr siècle aux peintres qui s'attachèrent à réaliser des vues (vedute) de villes.

2. Pour la définition et les attributs de l'icononaute, nous renvoyons à Gian Piero Brunetta (1997).

3. L'essai de Reclus, publié en 1895 et intitulé "The Evolution of Cities" dans Contemporary Review, fut présenté et traduit en français par Jean-Claude Chamboredon et Annie Méjean, "L'évolution des villes", dans Cahiers d'économies et sociologies rurales, $\mathrm{n}^{\circ} 8,1898$.

4. On trouve un imposant répertoire iconographique sur le Paris du tournant de siècle dans Juin, 1994.

5. La légende se lit comme suit "Basilique de Saint-Marc — "D'après: Calli et Canali, F. Ongania, Édit." ".

6. Dans l'ordre: Chiesa di S. Marco, 1841, incision au daguerréotype par N.P. Lerebours, Excursions daguerriennes, Paris, 1841, Venise, collection Costantini ; (Jean Walther?) Basilica di S. Marco, 1851, da L. D. Blanquart-Evrard, Album photographique de l'artiste et de l'amateur, Lille, 1851, Paris, B. N. ; Carlo Ponti, Basilica di S. Marco, 1860 env., estampes à l'albumine, Venise, Archives Böhm; Achille Quinet, Basilica di S. Marco, 1865, estampes à l'albumine, Paris, B. N., toutes reproduites dans Paolo Costantini et Italo Zannier, Venezia nella fotografia dell'Ottocento, Venezia, ArsenaleBöhm, 1896. On voit également la reproduction photographique de la fin du XIX siècle La Cathédrale de Saint-Marc. Venise, dans John L. Stoddard, Portfolio de photographies des villes, paysages et peintures célébres, s.d., Werner, Chicago.

7. Laffirmation de Promio - en particulier là où il soutient la possibilité de reproduire "grâce au cinéma mobile des objets immobiles" - fait référence aux considérations suscitées par l'intérêt partiel des vues Lumières pour la représentation spécifiquement architecturale.

8. Jacques Aumont définit quatre différents points de vue relatifs au langage cinématographique (Aumont, 1983).

9. Par exemple, Sandro Bernardi (1993, p. 15) met en évidence le "le fort coefficient d'autoreprésentation de cette ville [Paris] qui, lorsqu'elle se trouve devant l'appareil cinématographique, se transforme en une scène théâtrale ". Notre traduction.

10. Selon Riccardo Redi (1986, p. 14) : "Les premiers opérateurs du cinéma étaient des photographes et la photographie du XIX siècle devait toujours être parfaitement nette: la différenciation des plans par un changement de la mise au point était une pratique inusitée, inconcevable même. "Notre traduction.

11. De Claude Monet, je rappelle deux versions de la Gare Saint-Lazare, une conservée au Louvre (1877) et l'autre au Fogg Art Museum de Cambridge (1877); Gustave Caillebotte, Pont de l'Europe, 1876, Genève, Musée du Petit Palais; Camille Pissarro, Place du Théâtre-Français à Paris ou L'Avenue de l'Opéra, 1898, huile sur toile, Reims, musée des Beaux-Arts de Reims; Mardi gras, soleil couchant, boulevard Montmartre, 1897, huile sur toile, Winterthur, Kunstmuseum Winterthur.

12. Wassily Kandinsky, Vor der Stadt, 1908, München, Städtische Galerie im Lenbachhaus; Umberto Boccioni, Officine a Porta Romana, 1908, Milano, Collection de la Banca Commerciale Italiana.

13. Théophile Steilen, Paysages d'usines, 1895 env., huile sur toile, Paris, musée d'Orsay.

14. Constantin Meunier, Au Pays noir, 1890 env., Paris, musée d'Orsay. 


\section{RÉFÉRENCES BIBLIOGRAPHIQUES}

Anonyme, 1904: "Nouvelles Conférences avec projections ", Le Fascinateur, 1904.

Aumont, 1983: Jacques Aumont, "Le point de vue", Communications, $\mathrm{n}^{\circ} 38,1983$, p. 3-29.

Aumont, 1984 : Jacques Aumont, "Du cinématographe aux symphonies urbaines. La ville dans le cinéma muet ", Temps libre, n" 11, 1984.

Bernardi, 1993: Sandro Bernardi, Introduzione alla retorica del cinema, Firenze, Le Lettere, 1993.

Brunetta, 1997 : Gian Piero Brunetta, Il Viaggio dell'icononauta dalla camera oscura di Leonardo alla luce dei Lumière, Venezia, Marsilio, 1997.

Camporesi, 1992: Piero Camporesi, Le belle contrade. Nascita del paesaggio italiano, Milano, Garzanti, 1992.

Coissac, 1925: Georges-Michel Coissac, Histoire du Cinématographe. De ses origines à nos jours, Paris, Éditions du Cinéopse, Libraire Gauthier-Villars, 1925.

Costa, 1981 : Antonio Costa, "Paesaggio e cinema", dans T. Maldonado (dir.), Paesaggio: immagine e realtà, Milano, Electa, 1981.

De Seta, 1982 : Cesare De Seta, "L'Italia nello specchio del "Grand Tour" ", dans Storia d'Italia, Annali $V$, "Il paesaggio ", Torino, Einaudi, 1982.

Gardies, 1995: André Gardies, "La cité Lumière", CinémAction, n 75, Architecture, décor et cinéma, Françoise Puaux (dir.), 1995.

Gervereau, 1994: Laurent Gervereau, "Usines, faubourgs et prise de conscience ouvrière ", dans J. Dethier et A. Guilheux (dir.), La Ville. Art et architecture en Europe 1870-1993, Paris, centre Georges-Pompidou, 1994, p. 66-75.

Joanne, 1895: Paul Joanne, Venise, Paris, Hachette, 1895.

Juin, 1994: Hubert Juin, Le Livre de Paris 1900, Paris, Michèle Trinckvel, 1994.

Montani, 1989 : Pietro Montani, "Considerazioni estetiche sulla storia del cinema", Film Critica, $\mathrm{n}^{\circ} 400,1989$.

Natali, 1996: Maurizia Natali, L'Image-paysage. Iconologie et cinéma, Saint-Denis, Presses Universitaires de Vincennes, 1996.

Paquot et Roncayolo, 1992 : Thierry Paquot et Marcel Roncayolo (dir.), Villes et civilisation urbaine. XVIIT $-X X$ siècle, Paris, Larousse, 1992.

Pinel, 1974: Vincent Pinel, Louis Lumière, Paris, Anthologie de l'Avant-Scène, 1974.

Redi, 1986: Riccardo Redi (dir.), "Linvenzione di una macchina; o del cinema", Lumière. Verso il centenario, Roma, Di Giacomo, 1986.

Roger, 1999: Philippe Roger, «Le cinéma est-il né de la ville? Quand Lyon rencontrait le cinématographe ", dans L'Aventure du Cinématographe, Actes du Congrès mondial Lumière, Lyon. ALÉAS, 1999, p. 335-342.

Rondolino, 1988: Gianni Rondolino, Storia del cinema, Torino, UTET, 1988. 